\title{
Rapid and specific SPRi detection of L. pneumophila in complex environmental water samples
}

\author{
Amir M. Foudeh ${ }^{1} \cdot$ Hana Trigui $^{2}$ - Nilmini Mendis ${ }^{2}$ - Sebastien P. Faucher ${ }^{2}$. \\ Teodor Veres ${ }^{1,3} \cdot$ Maryam Tabrizian ${ }^{1}$
}

Received: 23 November 2014 /Revised: 13 March 2015 / Accepted: 17 April 2015 / Published online: 3 May 2015

(C) Springer-Verlag Berlin Heidelberg 2015

\begin{abstract}
Legionellosis is a very devastating disease worldwide mainly due to unpredictable outbreaks in man-made water systems. Developing a highly specific and sensitive rapid detection system that detects only metabolically active bacteria is a main priority for water quality assessment. We previously developed a versatile technique for sensitive and specific detection of synthetic RNA. In the present work, we further investigated the performance of the developed biosensor for detection of Legionella pneumophila in complex environmental samples, particularly those containing protozoa. The specificity and sensitivity of the detection system were verified using total RNA extracted from L. pneumophila in spiked water co-cultured with amoebae. We demonstrated that the expression level of ribosomal RNA (rRNA) is extremely dependent on the environmental conditions. The presence of amoebae with $L$. pneumophila, especially in nutritiondeprived samples, increased the amount of L. pneumophila 15 -fold after 1 week as measured through the expression of 16s rRNA. Using the developed surface plasmon resonance imaging (SPRi) detection method, we were also able to
\end{abstract}

Electronic supplementary material The online version of this article (doi:10.1007/s00216-015-8726-y) contains supplementary material, which is available to authorized users.

Maryam Tabrizian

maryam.tabrizian@mcgill.ca

1 Department of Biomedical Engineering, Faculty of Medicine, McGill University, 3775 University Street, Montreal, QC H3A 2B4, Canada

2 Department of Natural Resource Sciences, Faculty of Agricultural and Environmental Sciences, Macdonald campus, McGill University, 21111 Lakeshore, Sainte-Anne-de-Bellevue, QC H9X 3V9, Canada

3 National Research Council Canada, 75 Boul. de Mortagne, Boucherville, QC J4B 6Y4, Canada successfully detect L. pneumophila within $3 \mathrm{~h}$, both in the presence and absence of amoebae in the complex environmental samples obtained from a cooling water tower. These findings suggest that the developed biosensing system is a viable method for rapid, real-time and effective detection not only for L. pneumophila in environmental samples but also to assess the risk associated with the use of water contaminated with other pathogens.

Keywords Biosensors · Nanoparticles/nanotechnology · Nucleic acids (DNA $\mid$ RNA) · Optical sensors

\section{Introduction}

Legionella species are the causative agent of legionellosis, and among them, Legionella pneumophila is responsible for more than $90 \%$ of legionellosis. Legionellosis is a very devastating disease worldwide mainly due to unpredictable outbreaks. Legionellosis, which is transmitted through aerosol, is manifested as a form of pneumonia or Pontiac fever, a milder form of the disease with flu-like symptoms [1]. Between 2001 and $2006,30 \%$ of waterborne disease outbreaks in the USA were caused by Legionella [2]. The fatality rate of legionellosis can approach $50 \%$ within industrial and hospital outbreaks, especially affecting individuals with a compromised health condition [1]. L. pneumophila is found in most natural and engineered water systems, such as air conditioning, showers and cooling towers where it contaminates and multiplies inside amoebae [3].

Currently, L. pneumophila is mainly detected by laboratory culture, polymerase chain reaction (PCR), immunology-based methods and DNA microarray methods [4-6]. However, these detection methods all have shortfalls. The culture method is very time consuming and does not have the ability to detect 
viable but nonculturable cells (VBNC). PCR is unreliable in many situations, due to false-positive detection of nonviable bacteria and the presence of inhibitors in environmental water [7]. DNA microarrays are also unable to distinguish between live and dead bacteria. Targeting ribosomal RNA (rRNA) is a viable alternative that overcomes the aforementioned limitations: it provides a detection system that is more reliable, accurate and sensitive. This is due both to the correlation of the RNA expression level in bacteria with microbial activity and to the presence of high copy numbers of 16s rRNA in each bacterium.

We developed an effective technique for detection of synthetic RNA [8], through the design of specific DNA capture and detector probes along with the use of quantum dots (QDs) for signal amplification. We were able to detect sub-femtomole levels of synthetic RNA with the surface plasmon resonance imaging (SPRi) biosensor in less than $3 \mathrm{~h}$. Although the detection of synthetic RNA is the first step towards the development of a biosensor for on-site detection, the main challenge remains to validate the performance of the developed biosensor for much more complex situations such as the detection of RNA extracted from pathogenic $L$. pneumophila in environmental water samples, particularly when protozoa are present.

The interaction of protozoa, especially amoebae, with L. pneumophila in water systems is of great importance. Most of the conventional biosensors are unable to detect the $L$.pneumophila hidden inside amoebae and failed to provide any meaningful information regarding the interaction of Legionella with protozoa especially in the environmental water samples. L. pneumophila can normally survive in nutritiondeprived environments for long periods of time but cannot multiply. They multiply in these environments mostly when amoebae were also present [9]. The ingestion of L. pneumophila by amoebae provides an intra-cellular environment for its amplification in water systems. In addition, amoebae can also act as a shelter against harsh conditions such as low temperatures and the presence of biocides [9-12]. In the case of biocide treatment, this protection can result in treatment failure, after which $L$. pneumophila might be able to recolonize the water system rapidly. Another important impact of amoeba-Legionella interaction is the enhancement of the virulence of $L$. pneumophila [13]. It has been reported that their combined action contributes to L. pneumophila's virulence by priming the bacteria to infect human cells [14].

Therefore, in our current work, we investigated the interaction of the amoeba with $L$. pneumophila in the nutritiondeprived buffer and the environmental water samples. We further examined the specificity and sensitivity of our detection approach in these conditions and their effects on the biosensor performance with the ultimate goal of developing an on-site detection system (Scheme 1). In order to ensure specificity of the detection system, we first examined total RNA (totRNA) extracted from different bacteria and then the limit of detection of totRNA extracted from pathogenic L. pneumophila was

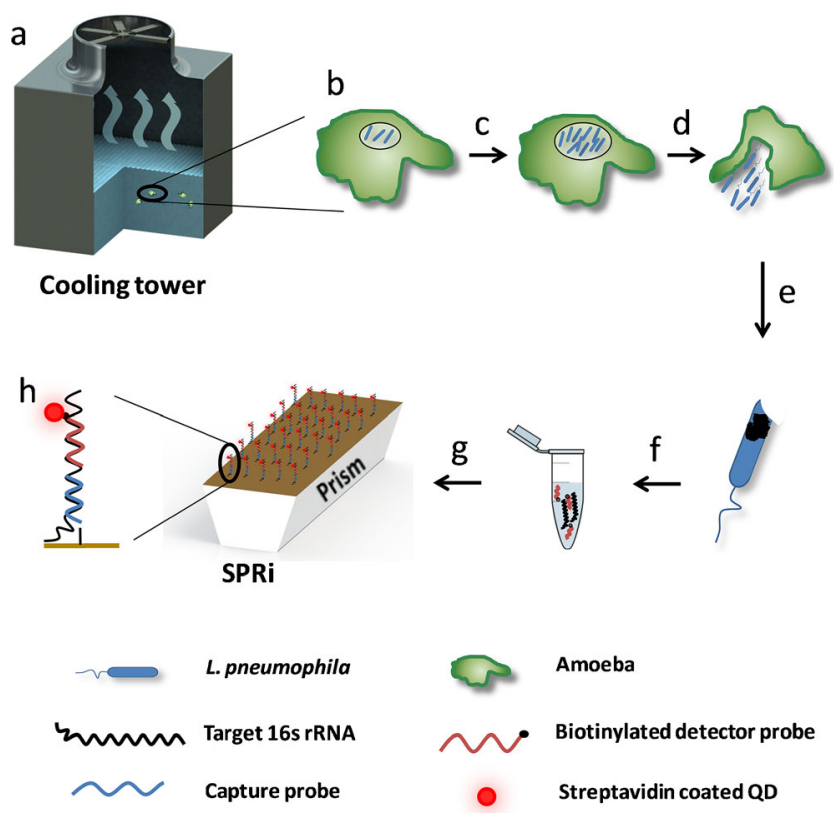

Scheme 1 Schematic illustration of the infection cycle of L. pneumophila in amoebae in cooling tower water and detection of L. pneumophila using SPRi: $a$ cooling tower water containing amoebae and $L$. pneumophila, $b$ an amoeba infected by L. pneumophila, $c$ multiplication of $L$. pneumophila inside an amoeba, $d$ lyses of amoeba and release of $L$. pneumophila, $e$ collection and lyses of $L$. pneumophila, $f$ extraction and fragmentation of RNA from L. pneumophila, $g$ hybridization of extracted RNA on the SPRi chip, $h$ schematic of the RNA hybridization using capture and detector probes and use of QDs post amplification

determined with our SPRi-based biosensor setup. In addition, the effect of residency of $L$. pneumophila in nutrition-deprived water samples and amoeba-Legionella interaction in a coculture system with defined water composition on 16s rRNA expression and on the SPRi signal at different time points was assessed. Finally, cooling tower water samples contaminated with $L$. pneumophila, in the presence and absence of amoebae, were examined to explore the viability of the developed technique for detecting $L$. pneumophila in a complex environment.

\section{Experimental}

"Materials and Methods" can be found in the Electronic Supplementary Material.

\section{Results and discussion}

\section{Assessment of specificity and sensitivity of the SPRi biosensor}

In order to evaluate the specificity of the detection system, the change in SPRi reflectivity $(\Delta \% \mathrm{R})$ of totRNA hybridization 
from $10^{6}$ colony-forming units $(\mathrm{CFU}) / \mathrm{mL}$ of $L$. pneumophila, two different strains of Escherichia coli (DH5 $\alpha$ and K12) and Pseudomonas aeruginosa were measured. As shown in Fig. S1, hybridization of totRNA extracted from all bacteria except for L. pneumophila did not result in a significant SPR signal. This confirmed that the designed capture and detector probes allowed for highly specific detection of $L$. pneumophila. To determine the sensitivity and limit of detection (LOD) for totRNA, a dilution series of L. pneumophila in AYE medium ranging from $3 \times$ $10^{4}$ to $3 \times 10^{8} \mathrm{CFU} / \mathrm{mL}$ was made, and $1 \mathrm{~mL}$ of each sample was used for RNA extraction. The extracted RNA was then fragmented and the hybridization kinetic was monitored in real time with a SPRi biosensor, employing the SA-QD signal amplification. The results indicated that RNA could be extracted from very low concentrations of bacteria, ranging from $3 \times 10^{4}$ to $3 \times 10^{8} \mathrm{CFU} / \mathrm{mL}$. A LOD comparable to that obtained for the detection of synthetic RNA [8] was achieved, thereby confirming the high sensitivity of the developed detection system in a complex mixture of RNA (Fig. S2).

\section{6s rRNA expression level}

The presence of L. pneumophila in nonoptimal conditions, especially in nutrition-deprived environments, has been reported to affect its metabolic activity which in turn influences the expression of 16s rRNA [15]. To investigate the metabolic activity of $L$. pneumophila in nutritiondeprived environments, L. pneumophila was incubated in AC buffer at different time points from 0 to $48 \mathrm{~h}$. Reverse transcriptase PCR was first performed to convert RNA to cDNA, and then real-time PCR was carried out to quantify the expression level of $16 \mathrm{~s}$ rRNA. Since in real-time $\mathrm{PCR}$, the cycle threshold $(\mathrm{Ct})$ is defined as the number of cycles required for the signal to exceed the background level, the Ct value is inversely proportional to the amount of RNA in the sample (Fig. 1). It has been reported that L. pneumophila cannot grow in AC buffer [16], and we further confirmed this by CFU counting for each sample (data not shown). Our results suggest that, even after $6 \mathrm{~h}$ of exposure of L. pneumophila to AC buffer, the level of 16s rRNA expression dropped significantly and this trend continued up to $48 \mathrm{~h}$ (Fig. 1). This further shows that the metabolic activity of bacteria is extremely dependent on their milieu, and confirms that targeting 16s rRNA in bacteria could give meaningful insight into the metabolic state of bacteria.

\section{SPRi detection of $L$. pneumophila co-cultured with amoebae}

In order to investigate the effect of amoebae presence on L. pneumophila purulence, $1.5 \times 10^{6}$ amoebae were cocultured with $1.5 \times 10^{6} \mathrm{CFU}$ of L. pneumophila in $\mathrm{AC}$

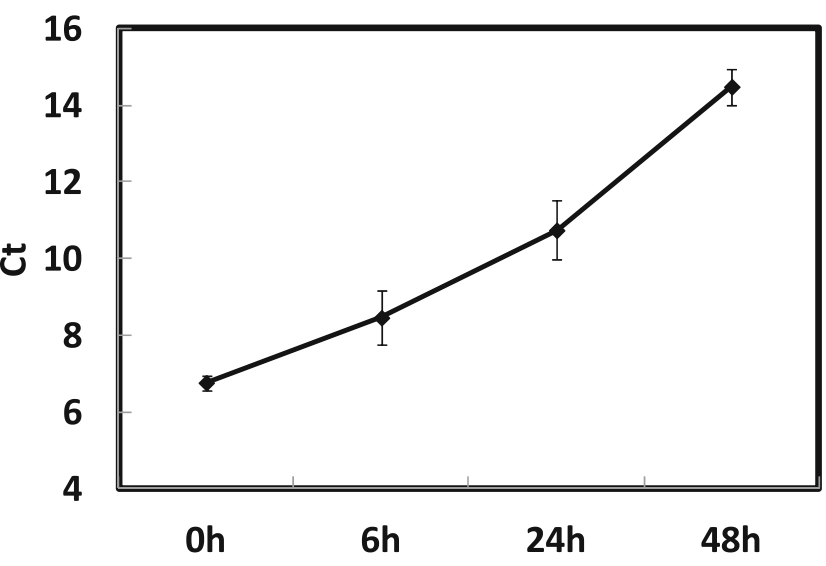

Fig. 1 The effect of incubation time of $L$. pneumophila in AC buffer on $16 \mathrm{~s}$ rRNA expression was examined. Ct values obtained from real-time PCR experiments and plotted against four different incubation time points. All data are expressed as mean \pm standard deviation

buffer. Figure 2 shows changes in SPRi signal as a function of L. pneumophila concentration after 1, 2 and 7 days in the presence and absence of amoebae. Interestingly for L. pneumophila in AC buffer, the SPRi signal dropped to $0.18 \pm 0.09 \Delta \% \mathrm{R}$ as of day 1 (Fig. $2 \mathrm{~b}$ ) which is significantly lower than at the same concentration in AYE $(2 \%$ change in reflectivity is expected at the same concentration in AYE, according to Fig. S2). This lower SPRi signal is obviously due to the reduction of $16 \mathrm{~s}$ RNA expression of L. pneumophila in a nutrient-poor medium as compared to the SPRi signal in an AYE medium. The drop in SPRi signal is also in agreement with our previous observation, depicted in Fig. 1. The $\mathrm{Ct}$ value for day 1 was significantly lower than that for day 0 . The SPRi signal for day 1 was stronger for the co-cultured samples than for the L. pneumophila cultured alone $(0.18 \pm 0.09 \Delta \% \mathrm{R}$ versus $0.72 \pm 0.13 \Delta \% \mathrm{R})$, while the CFU count remained the same for both (Fig. 2a). This further confirmed that the amoebae would enhance L. pneumophila $16 \mathrm{~s}$ rRNA expression.

In order to examine the effect of RNA extracted from amoebae on the detection system performance, the negative control samples containing only amoebae were also tested at all time points. No signals for amoeba samples were observed (data not shown). As seen in Fig. 2a, although the concentration of L. pneumophila in AC buffer remained the same from days 1 to 7 , the presence of amoebae in co-culture samples resulted in a significant increase of $L$. pneumophila concentration after $2(6.37 \pm$ $0.10 \mathrm{Log} C F U / \mathrm{mL})$ and 7 days $(7.64 \pm 2.24 \mathrm{Log} C F U / \mathrm{mL})$ as compared to day $1(6.11 \pm 0.17 \mathrm{Log} \mathrm{CFU} / \mathrm{mL})$. The same trend could be observed with SPRi results. The reflectivity change for the co-culture sample increased with incubation time. We believe that the increase in the SPRi signal is mainly due to the increase of $L$. pneumophila concentration and partly due to the increased expression of 16s rRNA. 
a

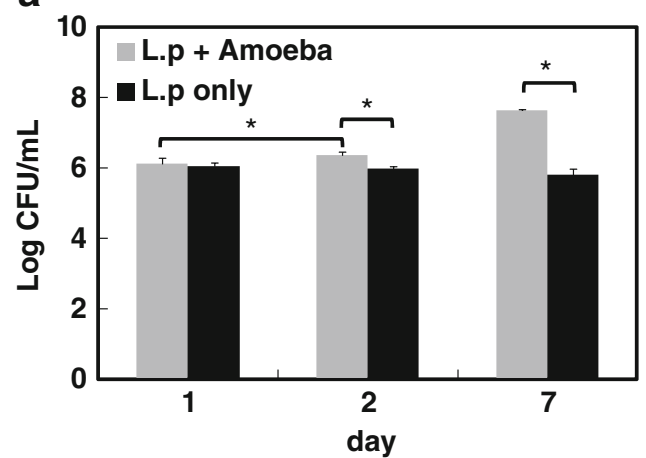

Fig. 2 Incubation of L. pneumophila in AC buffer in the presence and absence of amoeba after 1,2 and 7 days. a Concentration of L. pneumophila versus incubation time, b SPRi measurements of the hybridization of extracted RNA from $1 \mathrm{~mL}$ of each sample with QDs

\section{Validation of sensing technique for the cooling tower water sample}

To demonstrate the specificity and sensitivity of the system for the detection of L. pneumophila in complex environmental samples, L. pneumophila with a concentration ranging from $2 \times 10^{4}$ to $2 \times 10^{8} \mathrm{CFU} / \mathrm{mL}$ was spiked in a water sample from a cooling tower. A series of the SPRi measurements were performed in the presence and absence of amoebae after 2 days to assess the effect of this complex water sample on the L. pneumophila purulence and the 16s rRNA expression. As shown in Fig. 3a, the concentrations of L. pneumophila samples did not change after 2 days (1:1 linear correlation between days 0 and 2) while a significant increase of $L$. pneumophila concentration was observed when L. pneumophila was co-cultured with amoebae for all initial concentrations used in this study. After day 2, the increase in L. pneumophila concentrations in the co-culture samples was greater for the initial concentrations of 4, 5.3 and $6.2 \mathrm{Log} \mathrm{CFU} / \mathrm{mL}$ than for the initial concentrations of 7.1, 7.4 and 8.2 Log CFU/ $\mathrm{mL}$. This could be due to the difference in the infection ratio of $L$. pneumophila to amoebae. Since the initial

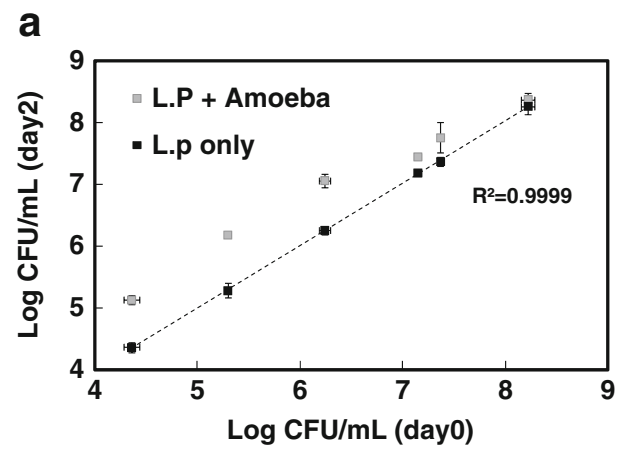

Fig. 3 Incubation of a dilution series of L. pneumophila in a cooling water sample in the presence and absence of $1.5 \times 10^{6}$ amoeba for 2 days. a CFU plate counting for each sample. b SPRi signal b

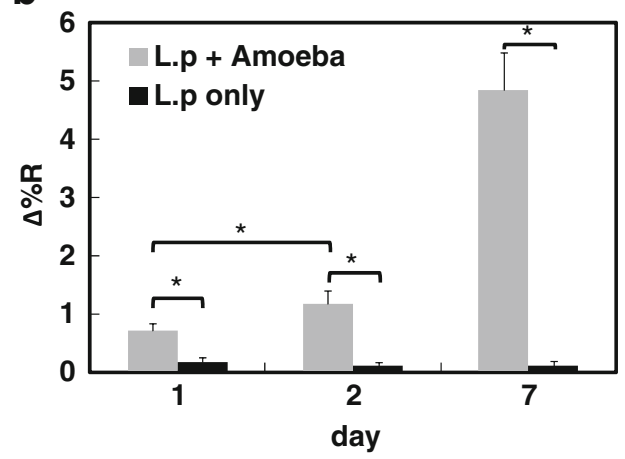

post amplification. An initial concentration of $10^{6} \mathrm{CFU}$ of L. pneumophila in the presence and absence of $10^{6}$ amoebae in a sixwell plate was used. All data expressed as mean \pm standard deviation $(* P<0.05)$

amoebae concentration was chosen as $6.2 \mathrm{Log}$ amoebae per sample, the infection ratio of less than one (samples with initial concentrations of 4, 5.3 and 6.2 Log CFU/mL) resulted in a more pronounced increase in concentration of L. pneumophila. This result is in agreement with literature reporting that at a higher infection ratio (when there are more bacteria per amoeba), the amoebae are lysed more rapidly [17, 18]. Therefore, there would be less amoebae for L. pneumophila to grow in, which would explain the reason behind our overall observation.

The presence of $L$. pneumophila in cooling tower water samples resulted in a higher SPRi signal as compared to signals from AC buffer samples shown in Fig. 3b. For instance, the sample with a concentration of 6.2 and 5.3 Log CFU $/ \mathrm{mL}$ resulted in $0.499 \pm 0.02 \Delta \% \mathrm{R}$ and $0.17 \pm$ $0.02 \Delta \% \mathrm{R}$ changes in reflectivity, respectively. These reflectivity changes were higher than the reflectivity change of $0.12 \pm 0.05 \Delta \% \mathrm{R}$ obtained from $6 \mathrm{Log} \mathrm{CFU} /$ $\mathrm{mL}$ in AC buffer after 2 days (Fig. 2b). This could be explained by the fact that the cooling tower water sample might contain more nutrition elements than the AC buffer. This higher concentration of nutrients can enhance the metabolic activity of L. pneumophila and therefore the

b

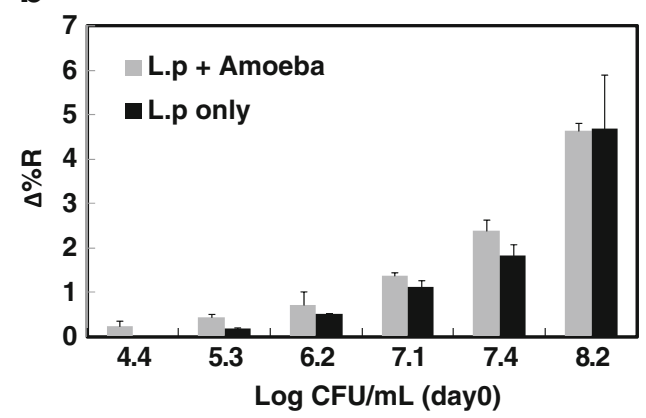

measurements of the hybridization of extracted RNA from $1 \mathrm{~mL}$ of each sample with QDs post amplification. All data expressed as mean \pm standard deviation 
16s rRNA expression level. As such, we could successfully detect L. pneumophila samples in the presence of amoebae with initial L. pneumophila concentrations as low as $4.4 \mathrm{Log}$ CFU/mL (Fig. 3b).

\section{Conclusions}

Monitoring metabolically active bacteria rapidly with high specificity and sensitivity is the main challenge in water quality assurance to prevent any potential outbreaks due to contaminated water systems. Using total RNA extracted from L. pneumophila along with SPRi technology, we investigated RNA as a viable genetic moiety that can provide a highly specific and sensitive detection modality for the detection of L. pneumophila in environmental water samples. We demonstrated that targeting 16s rRNA in L. pneumophila gives meaningful insight into the metabolic state of the bacteria by exposing bacteria to a nutrition-deprived environment and monitoring the change in $16 \mathrm{~s}$ rRNA expression with time. Our results showed that after only $6 \mathrm{~h}$ of exposure of L. pneumophila to a nutrition-deprived environment, the $16 \mathrm{~s}$ rRNA expression level decreased significantly. Interestingly, the presence of amoebae with $L$. pneumophila, in nutritiondeprived AC buffer, enhanced the expression of 16s rRNA after 1 day and resulted in a 15 -fold increase in L. pneumophila concentration after 1 week. Further development of this biosensing approach for detection of L. pneumophila would certainly contribute to the implantation of tools and platform for rapid, real-time and multiplex detection of bacteria, which is essential for water risk assessment of various sources.

Acknowledgments We acknowledge the National Science and Engineering Research Council of Canada-Strategic and Discovery programme, Genome Canada/Genome Quebec and Nano-Quebec. The authors would also like to thank R. Tien Sing Young for helping with illustrations design, L. Li for the amoeba culture, Dr. K. Bowey for her comments on the manuscript and Dr. Sandra Imbeault for providing the environmental water samples. The work in the SPF laboratory is supported by NSERC Discovery Grant 418289-2012.

\section{References}

1. Swanson M, Hammer B (2000) Legionella pneumophila pathogenesis: a fateful journey from amoebae to macrophages. Ann Rev Microbiol 54(1):567-613

2. Craun GF, Brunkard JM, Yoder JS, Roberts VA, Carpenter J, Wade T, Calderon RL, Roberts JM, Beach MJ, Roy SL (2010) Causes of outbreaks associated with drinking water in the United States from 1971 to 2006. Clin Microbiol Rev 23(3):507-528
3. Wadowsky R, Wilson T, Kapp N, West A, Kuchta J, Dowling J, Yee R (1991) Multiplication of Legionella spp. in tap water containing Hartmannella vermiformis. Appl Environ Microbiol 57(7):1950-1955

4. Lazcka O, Campo FJD, Muñoz FX (2007) Pathogen detection: a perspective of traditional methods and biosensors. Biosens Bioelectron 22(7):1205-1217

5. Foudeh AM, Fatanat Didar T, Veres T, Tabrizian M (2012) Microfluidic designs and techniques using lab-on-a-chip devices for pathogen detection for point-of-care diagnostics. Lab Chip 12(18):3249-3266

6. Deisingh AK, Thompson M (2002) Detection of infectious and toxigenic bacteria. Analyst 127(5):567-581

7. Stinear T, Matusan A, Hines K, Sandery M (1996) Detection of a single viable Cryptosporidium parvum oocyst in environmental water concentrates by reverse transcription-PCR. Appl Environ Microbiol 62(9):3385-3390

8. Foudeh AM, Daoud JT, Faucher SP, Veres T, Tabrizian M (2014) Sub-femtomole detection of 16 s rRNA from Legionella pneumophila using surface plasmon resonance imaging. Biosens Bioelectron 52:129-135

9. Borella P, Guerrieri E, Marchesi I, Bondi M, Messi P (2005) Water ecology of Legionella and protozoan: environmental and public health perspectives. Biotechnol Annu Rev 11:355-380

10. Barbaree JM, Fields BS, Feeley JC, Gorman GW, Martin WT (1986) Isolation of protozoa from water associated with a legionellosis outbreak and demonstration of intracellular multiplication of Legionella pneumophila. Appl Environ Microbiol 51(2): 422-424

11. Dupuy M, Mazoua S, Berne F, Bodet C, Garrec N, Herbelin P, Ménard-Szczebara F, Oberti S, Rodier M-H, Soreau S, Wallet F, Héchard Y (2011) Efficiency of water disinfectants against Legionella pneumophila and Acanthamoeba. Water Res 45(3): 1087-1094

12. Bouyer S, Imbert C, Rodier M-H, Héchard Y (2007) Long-term survival of Legionella pneumophila associated with Acanthamoeba castellanii vesicles. Environ Microbiol 9(5):13411344

13. Cirillo JD, Falkow S, Tompkins LS (1994) Growth of Legionella pneumophila in Acanthamoeba castellanii enhances invasion. Infect Immun 62(8):3254-3261

14. Molmeret M, Horn M, Wagner M, Santic M, Abu Kwaik Y (2005) Amoebae as training grounds for intracellular bacterial pathogens. Appl Environ Microbiol 71(1):20-28

15. Gourse RL, Gaal T, Bartlett MS, Appleman JA, Ross W (1996) rRNA transcription and growth rate-dependent regulation of ribosome synthesis in Escherichia coli. Annu Rev Microbiol 50(1): 645-677

16. Neumeister B, Reiff G, Faigle M, Dietz K, Northoff H, Lang F (2000) Influence of Acanthamoeba castellanii on intracellular growth of different Legionella species in human monocytes. Appl Environ Microbiol 66(3):914-919

17. Ohno A, Kato N, Sakamoto R, Kimura S, Yamaguchi K (2008) Temperature-dependent parasitic relationship between Legionella pneumophila and a free-living amoeba (Acanthamoeba castellanii). Appl Environ Microbiol 74(14):4585-4588

18. Declerck P, Behets J, Delaedt Y, Margineanu A, Lammertyn E, Ollevier F (2005) Impact of non-Legionella bacteria on the uptake and intracellular replication of Legionella pneumophila in Acanthamoeba castellanii and Naegleria lovaniensis. Microb Ecol 50(4):536-549 